\title{
Nomogram for predicting pathologic complete response after transarterial chemoembolization in patients with hepatocellular carcinoma
}

\author{
Jian Lin ${ }^{1 \#} \wedge$, Xiaowei Li ${ }^{2 \#}$, Xiaodong Shi ${ }^{3 \#}$, Lei Zhang ${ }^{1}$, Hongzhi Liu ${ }^{3}$, Jingfeng Liu ${ }^{4}$, Kui Wang ${ }^{1}$, Feng Shen ${ }^{1}$ \\ ${ }^{1}$ Department of Hepatic Surgery, Eastern Hepatobiliary Surgery Hospital, Second Military Medical University, Shanghai, China; ${ }^{2}$ Intervention \\ Center, Eastern Hepatobiliary Surgery Hospital, Second Military Medical University, Shanghai, China; ${ }^{3}$ Department of Hepatopancreatobiliary \\ Surgery, Mengchao Hepatobiliary Hospital of Fujian Medical University, Fuzhou, China; ${ }^{4}$ Department of Hepatopancreatobiliary Surgery, Cancer \\ Hospital of Fujian Medical University, Fuzhou, China \\ Contributions: (I) Conception and design: J Liu, K Wang, F Shen; (II) Administrative support: J Liu, K Wang, F Shen; (III) Provision of study \\ materials or patients: J Lin, L Zhang, H Liu; (IV) Collection and assembly of data: J Lin, L Zhang, H Liu; (V) Data analysis and interpretation: J Lin, \\ L Zhang, H Liu; (VI) Manuscript writing: All authors; (VII) Final approval of manuscript: All authors. \\ \#These authors contributed equally to this work. \\ Correspondence to: Feng Shen; Kui Wang. Department of Hepatic Surgery, Eastern Hepatobiliary Surgery Hospital, Second Military Medical \\ University, No. 225, Changhai Road, Shanghai 200438, China. Email: shenfengehbh@sina.com; wangkuiykl@163.com; Jingfeng Liu. \\ Department of Hepatopancreatobiliary Surgery, Cancer Hospital of Fujian Medical University, No. 420, Fuma Road, Fuzhou 350014, China. \\ Email: drjingfeng@126.com.
}

Background: The pathologic responses to transarterial chemoembolization (TACE) in patients with hepatocellular carcinoma (HCC) are heterogeneous and result in disparate outcomes. The study aimed to establish and validate an effective nomogram for predicting pathologic complete response (PCR) after TACE.

Methods: We analyzed the clinicopathologic characteristics of HCC patients undergoing hepatectomy following TACE. Variables with statistical significance in a multivariate logistic regression analysis were incorporated in the nomogram.

Results: We detected PCR in 64 (50.4\%) patients in the training cohort and 18 (37.5\%) patients in the validation cohort. Univariable and multivariable logistic regression revealed that hepatitis $\mathrm{B}$ virus (HBV) DNA load $(\mathrm{P}=0.031)$, $\alpha$-fetoprotein (AFP, $\mathrm{P}=0.040)$, maximum tumor diameter $(\mathrm{P}=0.003)$, preoperative TACE session ( $\mathrm{P}=0.026$ ), and modified Response Evaluation Criteria in Solid Tumors (mRECIST) complete response $(\mathrm{P}=0.030)$ were identified as significant predictors of PCR. Incorporating these 5 factors, a nomogram was developed which attained concordance indexes of 0.80 [95\% confidence interval (CI): 0.720.87 ] and 0.82 (95\% CI: 0.68-0.95) for predicting PCR in the training and validation cohorts, respectively.

Conclusions: The easy-to-use nomogram achieved a good post-TACE prediction of PCR in HCC patients. Using the model, patients who would benefit most from TACE could be identified, and the subsequent appropriate procedures could be guided accordingly.

Keywords: Nomogram; hepatocellular carcinoma (HCC); pathologic complete response (PCR); transarterial chemoembolization (TACE)

Submitted Mar 09, 2021. Accepted for publication Jun 01, 2021.

doi: $10.21037 / \mathrm{atm}-21-1120$

View this article at: https://dx.doi.org/10.21037/atm-21-1120

\footnotetext{
$\wedge$ ORCID: 0000-0002-6928-9207.
} 


\section{Introduction}

Hepatocellular carcinoma (HCC) is a common malignancy and the second most frequent cause of cancer-related death worldwide (1). The European Association for the Study of the Liver (EASL) and American Association for the Study of Liver Diseases (AASLD) clinical guidelines recommend that liver resection, liver transplantation and, radiofrequency ablation should be considered in patients at Barcelona Clinic Liver Cancer (BCLC) stage A. Transarterial chemoembolization (TACE) is recommended as the first-line treatment for unresectable HCC, the bridge strategy for liver transplantation and downstaging therapy for curative hepatectomy with Child-Pugh class A or B and good performance (1-3). It can also be performed in HCC patients at BCLC stage A who are not feasible for surgery or ablation for reasons such as old age, tumor location, personal willingness, and so on (4).

A recent study reported that the local HCC response to the initial TACE correlates well with the efficacy of subsequent treatments, even the prognosis of the patients (5). Therefore, the accurate estimation of HCC response to TACE may be of great clinical significance, because it can evaluate the therapeutic efficacy and help surgeons choose appropriate subsequent procedures. Although TACE has been widely performed in clinical practice, the responses to TACE are heterogeneous and it is still unclear and controversial which group of patients has optimal HCC responses and benefit most from TACE (6). Histopathological examination is considered a gold standard for the evaluation of HCC response. Unfortunately, histopathological examination depends on surgical specimens that can only be obtained after liver resection or transplantation. Many clinical standards on the assessment of HCC response to TACE have been made over the past decade, including the World Health Organization (WHO) criteria (7) and modified Response Evaluation Criteria in Solid Tumors (mRECIST) guideline (8). However, such evaluation criteria mainly depend on imaging examination, sometimes leading to discrepancies when compared to pathological examination results. The accuracy of radiological examination may be significantly decreased by the dense lipiodol accumulation in HCC lesions after TACE, which may mask tumoral enhancement and make the detection of viable tumors difficult on contrastenhanced computed tomography (CT) (9). In addition, peritumoral inflammation resulting from TACE can also lead to false-positive diagnoses of tumoral necrosis on contrast-enhanced magnetic resonance imaging (MRI) (10). In previous studies, the sensitivity of contrast-enhanced CT was $36-72 \%$ and that of contrast-enhanced MRI was $43-85 \%$, in detecting residual viable HCC compared with the histopathologic examination $(11,12)$. Inaccurate evaluation of HCC response may contribute to detrimental consequences, especially misleading the clinician to select the wrong subsequent procedures.

Due to the lack of a reliable and convenient predictive method, developing a model for predicting pathologic complete response (PCR) after TACE in patients with HCC based on preoperative clinicopathologic data becomes desirable. Compared with other predictive models, a nomogram can provide an individualized, intuitive, easy-touse, and highly accurate estimation based on a combination of variables. The purpose of this study was to identify predictive factors associated with PCR after TACE in HCC patients. Furthermore, we aimed to establish and externally validate a nomogram by using readily available clinical variables to predict the possibility of PCR after TACE. We present the following article in accordance with the TRIPOD reporting checklist (available at https://dx.doi. org/10.21037/atm-21-1120).

\section{Methods}

\section{Patients}

In this study, we enrolled 2 independent cohorts of HCC patients who were treated with liver resection following TACE. The training and validation cohorts were composed of consecutive patients, and their data were retrospectively reviewed at the Eastern Hepatobiliary Surgery Hospital from October 2014 to December 2019 and at Mengchao Hepatobiliary Hospital of Fujian Medical University from June 2014 to April 2020, respectively. The study was conducted in accordance with the Declaration of Helsinki (as revised in 2013). The study was approved by the institutional ethics board of Eastern Hepatobiliary Surgery Hospital (No.: EHBHKY2020-K-028) and by the ethical review board of Mengchao Hepatobiliary Hospital of Fujian Medical University (No.: 2019-063-01). All patients gave written informed consent for their data to be used for research purpose.

The inclusion criteria were as follows: (I) HCC was preoperatively diagnosed using radiological tests (contrastenhanced CT or MRI of the abdomen) and postoperatively confirmed by pathological examinations; (II) BCLC stage 
A or B; (III) Child-Pugh A liver function; (IV) complete clinicopathologic data. Patients who received any preoperative anticancer treatments other than TACE were excluded.

\section{Preoperative examinations}

Demographics and preoperative examinations of patients included age, gender, routine blood test, liver function test, hepatitis B surface antigen (HBsAg), hepatitis B virus (HBV) DNA load, serum $\alpha$-fetoprotein (AFP) level, preoperative TACE session, presence of cirrhosis, maximum tumor size, tumor number and BCLC stage. The preoperative S index was calculated using gamma-glutamyl transferase (GGT), platelet (PLT) and albumin (ALB) [S-index: 1,000 × GGT/ $\left.\left(\mathrm{PLT} \times \mathrm{ALB}^{2}\right)\right]$. Measurement of maximum tumor diameter and tumor number were based on the preoperative contrastenhanced CT or MRI scan.

\section{Preoperative and postoperative response-evaluation}

Due to conventional TACE and drug-eluting bead TACE (DEB-TACE) having been reported to have equal effects in some large multicenter randomized controlled trials, all transarterial therapies in our study were categorized as TACE for analysis $(13,14)$. All preoperative imaging examinations were reviewed independently by 2 experienced radiologists who were blinded to patients' clinical data.

Dense compact lipiodolization with complete disappearance of any intratumoral enhancement on CT scan, or no enhancement of all target lesions on MRI was defined as the complete response of mRECIST (8). When the categorization of radiologic response was not definite, a final judgement was made via consensus of the 2 radiologists. Liver resection was performed at least 1 month after the most recent TACE. The indications and contraindications of hepatectomy were similar to the previous study (15). Two experienced hepatic pathologists, blinded to the preoperative clinical and radiologic data, independently measured the percentage necrosis of each target lesion, using the slice of the maximum diameter at $20 \times$ magnification on slides stained with hematoxylin and eosin (16). Any disagreements regarding the necrotic extent evaluation between the pathologists were settled by discussion, and a final standard pathologic report was generated. PCR was defined as $100 \%$ necrosis of each target lesion. Cirrhosis was confirmed by histopathological examination of the noncancerous part of the resected specimens according to the Meta-analysis of Histological
Data in Viral Hepatitis (METAVIR) classification (17). Microvascular invasion and its pathological grading were not available because they could not be evaluated in PCR.

\section{Statistical analysis}

Statistical analysis was conducted using SPSS software (version 25.0; IBM Corp., Armonk, NY, USA) and R project (version 4.0.2; R Foundation for Statistical Computing, Vienna, Austria). Continuous variables were expressed as mean (SD) and compared using two-tailed independent $t$-test. Categorical variables were presented as frequencies (percentages), and compared using the chi-square $\left(\chi^{2}\right)$ test or Fisher's exact test. Data in the training cohort were assessed by univariate logistic regression analysis for identifying variables associated with PCR. Multivariable logistic regression analyses were carried out on variables that reached $\mathrm{P}<0.05$ at univariate analysis. A $\mathrm{P}$ value $<0.05$ (two-tailed) was considered to indicate statistical significance for each analysis.

A nomogram was constructed based on the results of multivariable logistic regression analysis, using the rms package of $\mathrm{R}$. The nomogram has a reference line on the peak for scoring of each predictor from a 0 to 100 -point scale. The variables were displayed with a scale that visually revealed the relative weight and predicted probabilities of PCR. To quantify the predictive performance of the nomogram, concordance index (C-index) and calibration with 1,000 bootstrap samples were calculated to reduce the overfit bias (18). Generally, a C-index value higher than 0.75 represents relatively good discrimination.

\section{Validation of the nomogram}

The prediction model formed in the training cohort was applied to the validation cohort to validate and evaluate the predictive power of the nomogram. Based on the nomogram, the total scores of each patient were calculated. Then, receiver operating characteristic (ROC) curve analysis was performed to calculate the optimal cutoff value of the total scores, determined by the maximum value of the Youden index (i.e., sensitivity + specificity -1 ).

\section{Results}

\section{Clinicopatbologic characteristics}

127 and 48 HCC patients undergoing liver resection after 
TACE were consecutively enrolled into the training and validation cohorts during the study period, respectively. The baseline clinicopathologic characteristics of the training and validation cohorts are listed in Table 1. The majority of the baseline clinicopathologic data were similar between the 2 cohorts. The validation cohort had significantly larger tumor size and more tumor numbers. More advanced BCLC stage was more frequently observed in the validation cohort. PCR after TACE was found in $64(50.4 \%)$ and $18(37.5 \%)$ patients in the training and validation cohorts, respectively.

\section{Identification of prognostic factors of predicting PCR after TACE in HCC patients}

Univariate analysis revealed that low HBV-DNA $(\mathrm{P}=0.002)$, low AFP $(\mathrm{P}=0.004)$, small tumor diameter $(\mathrm{P}<0.001)$, multiple preoperative TACE sessions $(\mathrm{P}=0.041)$ and mRECIST complete response $(\mathrm{P}=0.007)$ were identified as significant predictive factors of PCR. In multivariate analysis, $\mathrm{HBV}$-DNA load $\leq 1 \times 10^{2} \mathrm{IU} / \mathrm{mL}$ [odds ratio (OR), 3.42; 95\% CI: $1.58-7.40 ; \mathrm{P}=0.002]$, AFP $\leq 20 \mathrm{ng} / \mathrm{mL}$ (OR, 3.06; 95\% CI: 1.44-6.50; P=0.004), maximum tumor diameter $\leq 5 \mathrm{~cm}(\mathrm{OR}, 4.48$; $95 \%$ CI: 1.52-7.96; $\mathrm{P}<0.001)$, multiple preoperative TACE sessions (OR, 2.37; 95\% CI: 1.04-5.44; $\mathrm{P}=0.041)$, and mRECIST complete response (OR, 2.79; 95\% CI: 1.32-5.89; $\mathrm{P}=0.007)$ were independently associated with PCR (Table 2).

\section{Development and validation of a PCR-predicting nomogram}

The predicting nomogram that contained all the independent predictive factors for PCR is shown in Figure 1. The C-index for PCR prediction in the training cohort was 0.80 (95\% CI: $0.72-0.87$ ). In addition, the calibration plot showed good agreement on PCR between the prediction by nomogram and the actual observation by histopathologic examination (Figure 2).

The nomogram also displayed a C-index of 0.82 (95\% CI, 0.68-0.95) for the estimation of PCR, and the calibration curve showed good consistency between prediction and observation in the probability of PCR in the validation cohort (Figure 3).

\section{Predictive accuracy of the PCR-predicting nomogram}

The optimal cut-off values of the total nomogram scores in training and validation cohorts were 169 and 149 , respectively. The sensitivity, specificity, positive predictive value, negative predictive value, positive likelihood ratio and negative likelihood ratio for predicting PCR were $85.9 \%, 60.3 \%, 68.4 \%, 81.0 \%, 2.2$ and 0.2 in the training cohort, and $83.3 \%, 76.7 \%, 78.1 \%, 82.1 \%, 3.6$ and 0.2 in the validation cohort, respectively.

\section{Discussion}

Current advice states that TACE is used as the first-line treatment for some HCC patients at BCLC stage A or B and can improve selected patients' prognosis $(19,20)$. It has already been found that PCR of HCC patients who underwent TACE strongly predict tumor-free survival after liver transplantation (21). However, the pathologic responses of HCC to TACE are substantially different, even at the same BCLC stage, which would render distinct survival outcomes $(5,6)$. Thus, there is an urgent need to develop precise predicting tools to evaluate pathologic response after TACE. Our study revealed that post-TACE factors, including HBV-DNA load $\leq 1 \times 10^{2} \mathrm{IU} / \mathrm{mL}$, AFP $\leq 20 \mathrm{ng} / \mathrm{mL}$, maximum tumor diameter $\leq 5 \mathrm{~cm}$, multiple TACE sessions and mRECIST complete response, are significantly associated with PCR.

Previously, a series of studies were carried out to predict the PCR by using preoperative clinical, laboratory and radiological data. The mRECIST, which was established a decade ago, is still extensively used to evaluate the therapeutic effect of solid tumors through radiological examination (8). However, in assessing HCC responses to TACE treatment, arterio-portal shunts, adjacent hepatic inflammation, coagulative hemorrhage, and the masking effect of lipiodol accumulation can influence arterial enhancement and make it difficult to diagnose the residual viable tumor $(12,22,23)$. Recently, a small sample-size study attempted to detect residual viable HCC based on measuring the density of iodine depositions after TACE (24). Unfortunately, lipiodol accumulation is affected by tumor vascularity and lipiodol uptake, which may limit its utility in clinical practice (25). In addition, response evaluation that depends merely on radiological examination may be influenced by the radiologists' subjective judgment, leading to misdiagnosis (26). Recently, Peng et al. (27) used residual convolutional neural network to develop a model for predicting response to TACE. Although the deep learning model had high accuracy, the study focused only on intermediate-stage HCC, and this model required specific 
Table 1 Baseline clinicopathologic characteristics in the training and validation cohorts

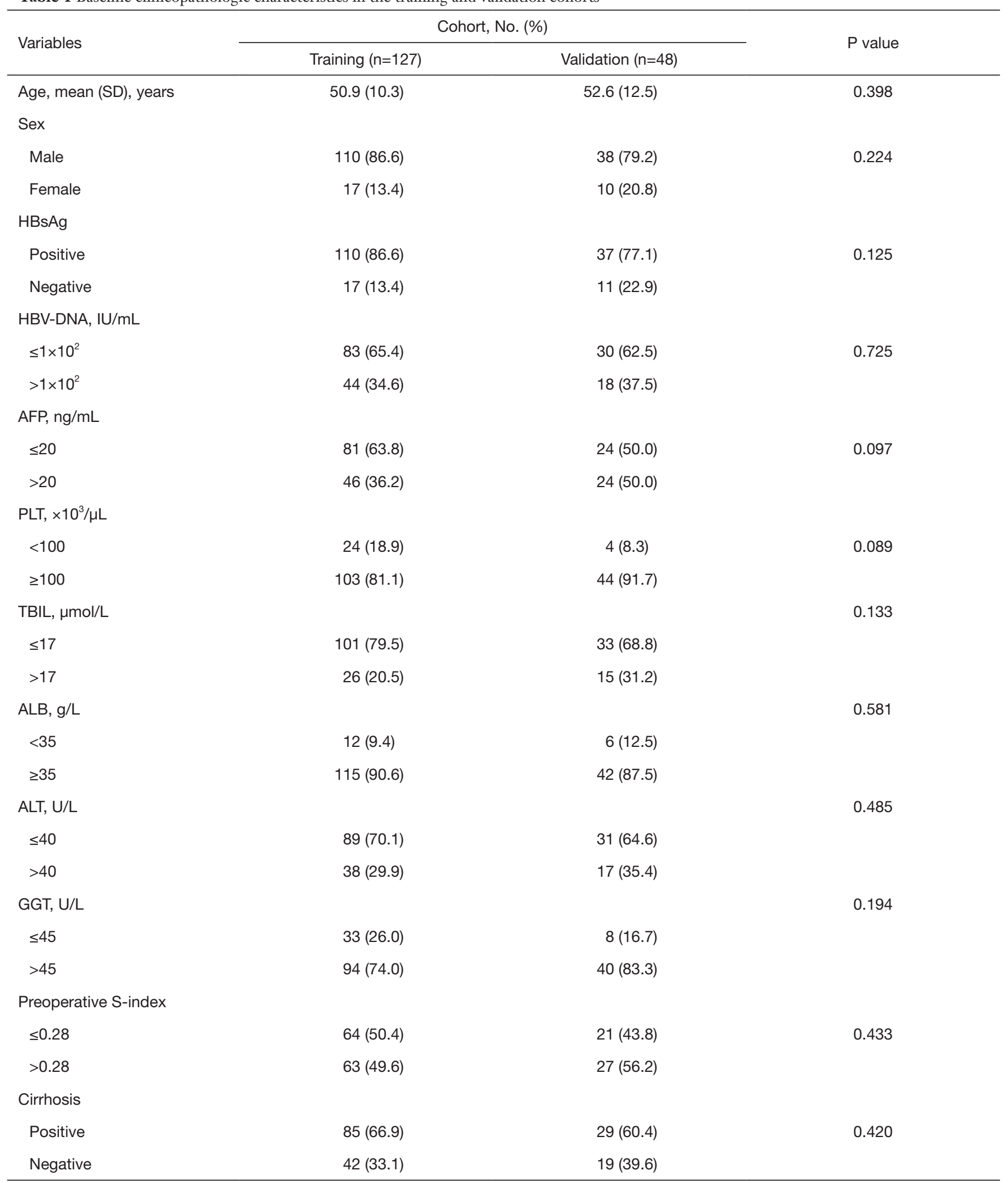

Table 1 (continued) 
Table 1 (continued)

\begin{tabular}{|c|c|c|c|}
\hline Variables & \multicolumn{2}{|c|}{ Cohort, No. (\%) } & $P$ value \\
\hline \multicolumn{4}{|c|}{ Maximum tumor diameter, $\mathrm{cm}$} \\
\hline$\leq 5$ & $61(48.0)$ & $11(22.9)$ & 0.003 \\
\hline$>5$ & $66(52.0)$ & $37(77.1)$ & \\
\hline Solitary & $108(85.0)$ & $30(62.5)$ & 0.001 \\
\hline Multiple & $19(15.0)$ & $18(37.5)$ & \\
\hline \multicolumn{4}{|c|}{ Preoperative TACE session } \\
\hline Single & $95(74.8)$ & $31(64.6)$ & 0.179 \\
\hline Yes & $64(50.4)$ & $18(37.5)$ & 0.127 \\
\hline No & $63(49.6)$ & $30(62.5)$ & \\
\hline \multicolumn{4}{|c|}{ mRECIST complete response } \\
\hline Yes & $48(37.8)$ & $20(41.7)$ & 0.639 \\
\hline No & $79(62.2)$ & $28(58.3)$ & \\
\hline \multicolumn{4}{|c|}{ BCLC Stage, n (\%) } \\
\hline A & $112(88.2)$ & $30(62.5)$ & 0.000 \\
\hline$B$ & $15(11.8)$ & $18(37.5)$ & \\
\hline
\end{tabular}

SD, standard deviation; HBsAg, hepatitis B surface antigen; HBV, hepatitis B virus; AFP, $\alpha$-Fetoprotein; PLT, platelet; TBIL, total bilirubin; ALB, albumin; ALT, alanine aminotransferase; GGT, $\gamma$-glutamyltransferase; TACE, transarterial chemoembolization; mRECIST, modified Response Evaluation Criteria in Solid Tumors; BCLC, Barcelona Clinic Liver Cancer.

computer software which would limit its use in clinical practice. The nomogram developed in the present study consists of 5 easily available preoperative variables from laboratory and imaging examinations, and medical history of treatment. The present nomogram performed well with a C-index of 0.80 in the training and 0.82 in the validation cohort. The calibration curves for the likelihood of PCR demonstrated optimal agreement between nomogram prediction and actual observation. The nomogram's predictive performance was also confirmed as remarkable in the external validation cohort.

Besides the classical mRECIST, HBV-DNA load, AFP, maximum tumor diameter, and TACE session were incorporated into the nomogram. AFP has been reported to be significantly correlated with PCR in HCC patients with cirrhosis after TACE (28). Another study showed that AFP response following TACE correlated well with radiological response in intermediate-stage HCC (29). Golfieri et al. (30) investigated the relationship between tumor size and the extent of tumor necrosis induced by TACE and observed an increase percentage of necrosis in larger lesions, which we hypothesize was associated with more developed arterial neoangiogenesis in such tumors. The present study also demonstrated a close association between tumor diameter and PCR, which was similar to the findings of some recent studies $(28,31,32)$. In previous clinical studies $(33,34)$, multiple TACE sessions were often needed, as the best response cannot always be attained after a single TACE session, particularly in large tumors. Such findings were consistent with those of the current study.

Although there was no report on the association between HBV DNA load and PCR, a few studies have suggested a 
Table 2 Univariable and multivariable analyses of variables associated with pathologic complete response in the training cohort

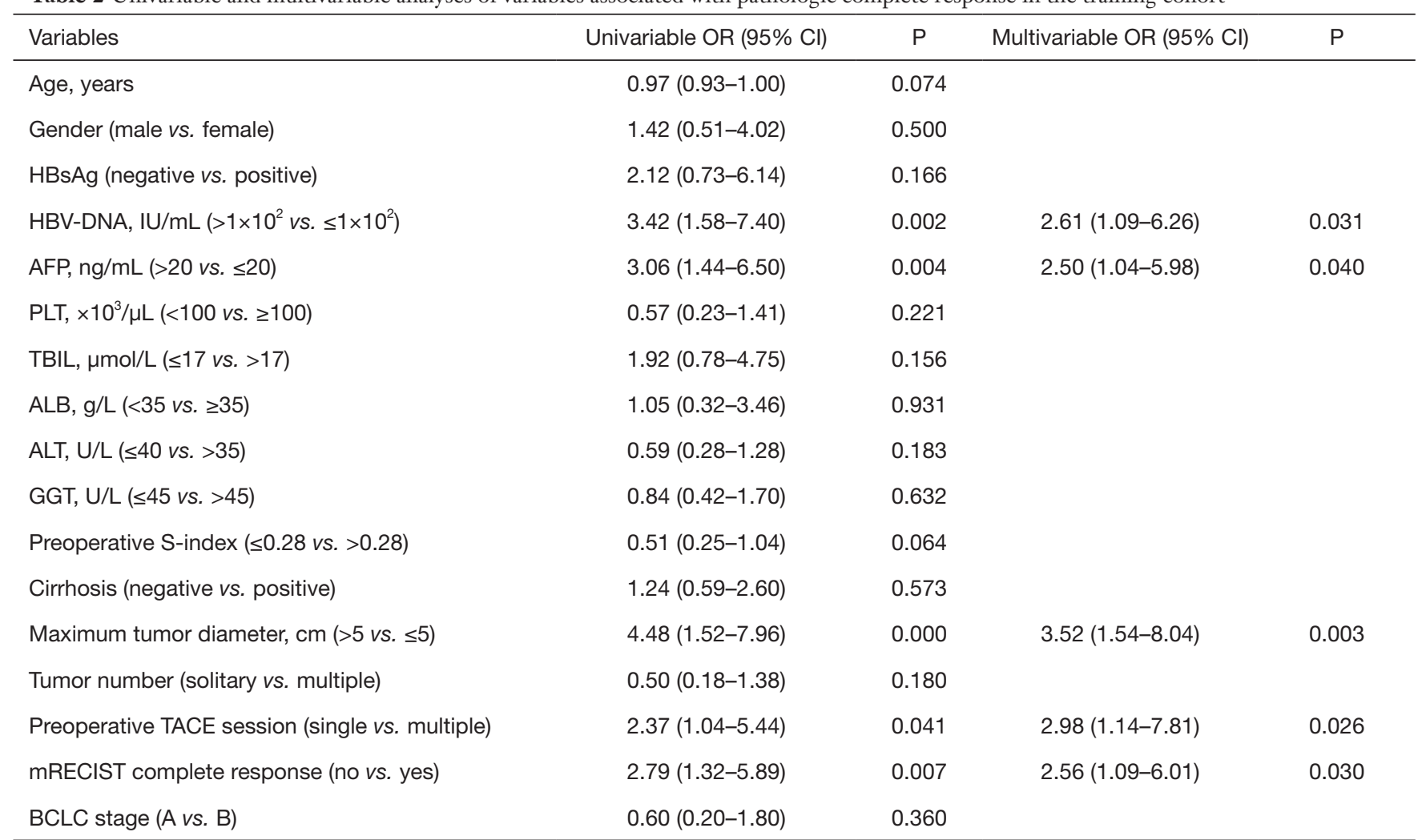

OR, odds ratio; Cl, confidence interval; HBsAg, hepatitis B surface antigen; HBV, hepatitis B virus; AFP, $\alpha$-Fetoprotein; PLT, platelet; TBIL, total bilirubin; ALB, albumin; ALT, alanine aminotransferase; GGT, $\gamma$-glutamyltransferase; TACE, transarterial chemoembolization; mRECIST, modified Response Evaluation Criteria in Solid Tumors; BCLC, Barcelona Clinic Liver Cancer.

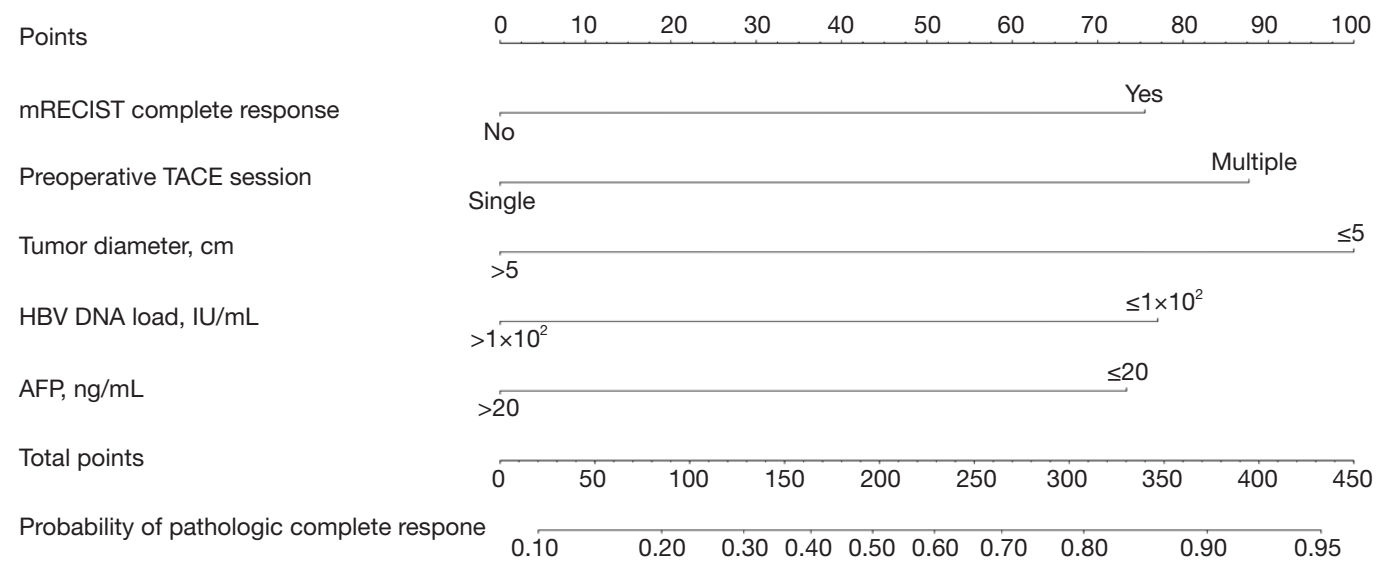

Figure 1 Nomogram to preoperatively estimate the probability of pathologic complete response after transarterial chemoembolization in patients with hepatocellular carcinoma. To use this nomogram, find the position of individual variable of each patient on the corresponding axis, draw a line upward to the points axis for the number of points of relevant variable, then accumulate all the points of an individual patient, and draw a line downward from the total points to the lowest line to determine the probability of pathologic complete response. 

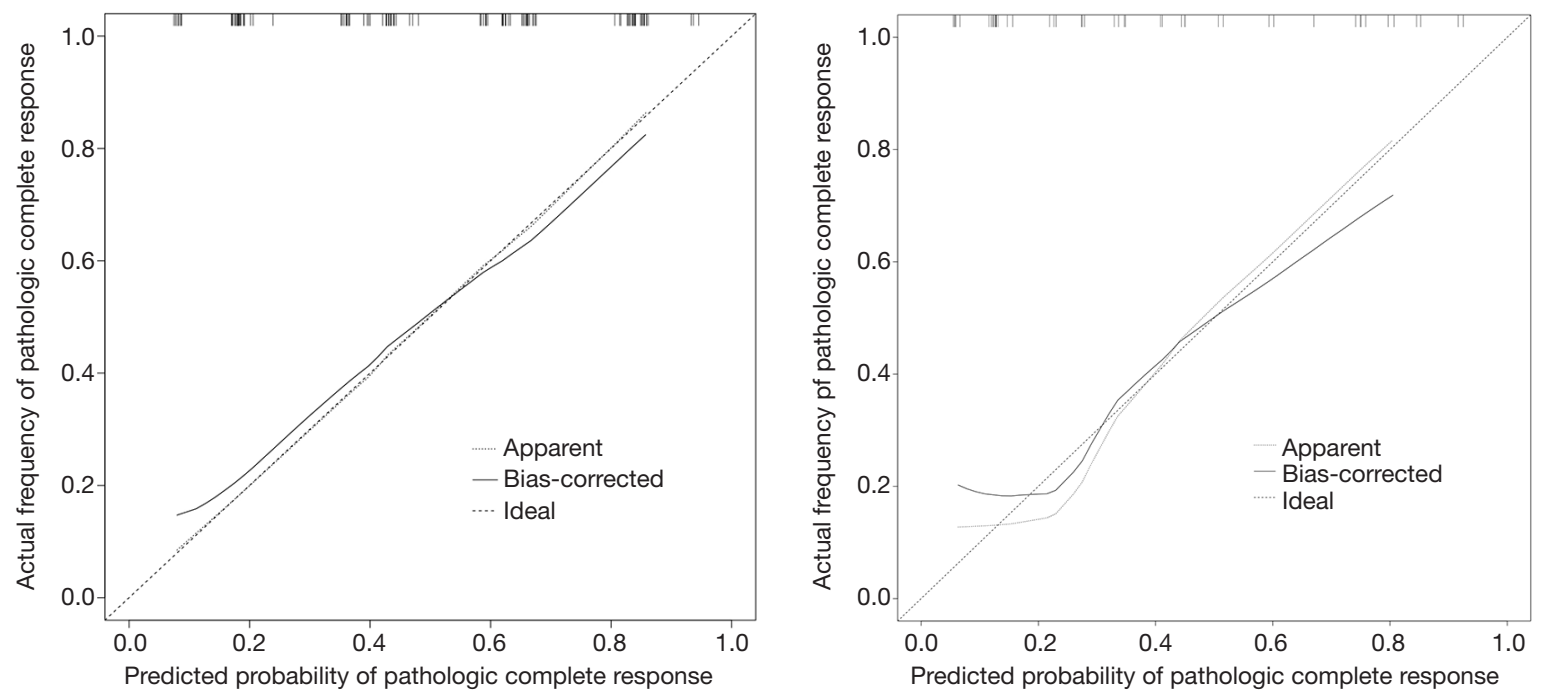

Figure 2 Calibration curves of the nomogram in the training and validation cohorts. The $\mathrm{X}$-axis is the probability of pathologic complete response predicted by the nomogram, and the Y-axis represents the actual probability. The long-dashed diagonal line shows an ideal nomogram with a perfect match with the actual observation. The bias-corrected performance of our nomogram is represented by the solid line, and the apparent accuracy of the present nomogram is showed by the short-dashed line.
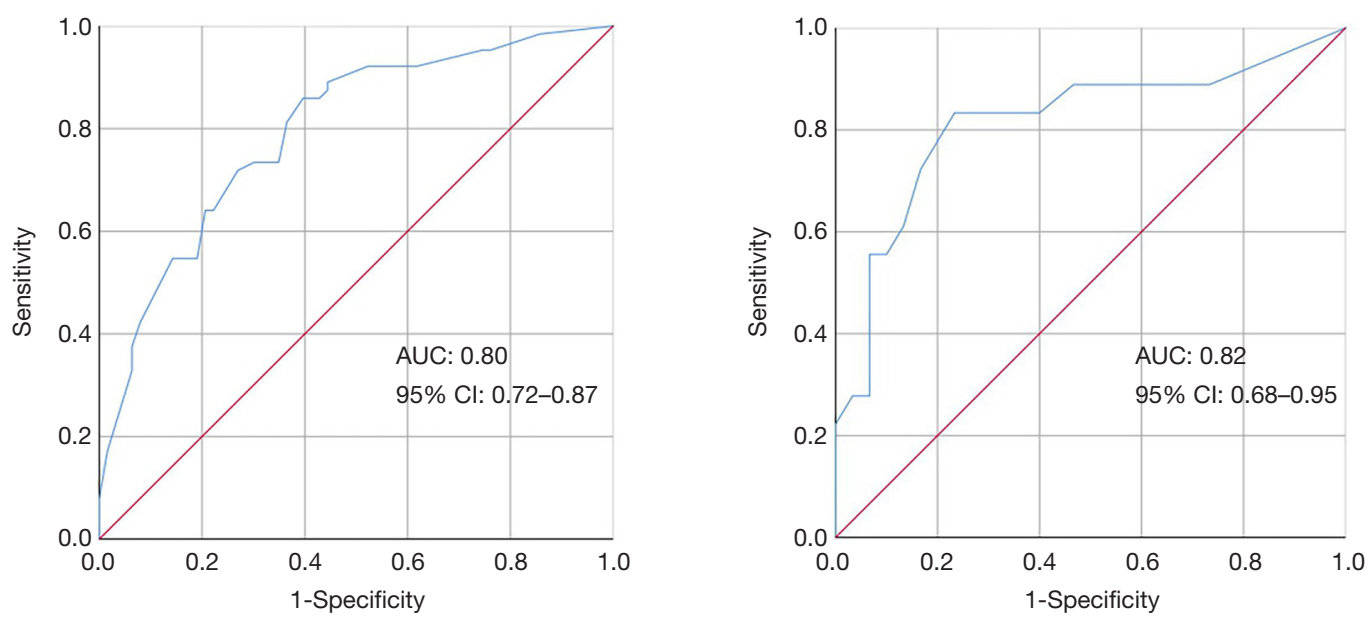

Figure 3 Receiver operating characteristic (ROC) curves of the nomogram in the training and validation cohorts. The ROC curves for the prediction of pathologic complete response depends on the logistic regression model. The area under the curve (AUC) in the training and validation cohorts are 0.80 (95\% CI, 0.72-0.87) and 0.82 (95\% CI, 0.68-0.95), respectively.

relationship between these 2 factors. Su et al. (35) found that elevated HBV DNA correlated well with lipiodol retention after TACE, and incomplete lipiodol deposition was considered to be an indication of substantial viable tumor remnant (36). Given the association above, we speculated that HBV DNA load might impact HCC response to TACE by influencing lipiodol deposition. However, further research is required to explore the underlying mechanism and validate our speculation.

The clinical decision-making, such as repeated TACE, liver transplantation, hepatectomy or follow-up observation, mainly depended on the response to initial TACE treatment. Our intuitive and easy-to-use nomogram can serve as a tool to accurately evaluate the pathologic response and 
thus carries significant clinical implications. As described in recent studies $(37,38)$, researchers have reported that repeated TACE cannot provide a markedly higher survival benefit in some patients, and ineffective TACE may even lead to detrimental effects. The serum concentration of vascular endothelial growth factor (VEGF) increases substantially after TACE and remains at a high level for at least 4 weeks (39). It promotes vasodilation and splanchnic vascularization, aggravates hyperdynamic splanchnic circulation, eventually increases portal pressure, and even leads to liver fibrosis progression (40). Additionally, TACE also induces ischemic injury in surrounding normal liver tissue (41). These unfavorable factors affect hepatocyte regeneration, suppress tumor immunity and deteriorate liver function (42). Elevated VEGF also contributes to the angiogenesis of HCC, which is responsible for tumor progression (43). Our nomogram can identify candidate patients who are more likely to achieve PCR, avoiding futile and potentially harmful repeated TACE. PCR has also been associated with excellent outcomes in HCC patients who received preoperative TACE and liver transplantation (28). Optimal candidates for liver transplantation can be selected by using the nomogram, and a good prognosis may be expected.

Although traditionally regarded as a non-curative treatment, TACE remains a promising procedure. Luo et al. (44) found no significant difference in prognosis between patients who underwent subsequent hepatic resection and those who did not, when those patients had responded well to initial TACE. Another study compared the patients' outcomes of superselective TACE and hepatic resection for resectable early-stage HCC, and observed that they had similar overall survival rates (45). A propensity score matching analysis reported that hepatectomy and TACE appeared to be equivalently effective for patients with solitary $\mathrm{HCC} \leq 5 \mathrm{~cm}$ (46). A previous clinical trial also showed that of 126 patients with PCR, only 3 developed recurrences and there was only 1 cancer-related death after liver transplantation; therefore, the authors concluded that PCR was tantamount to curative treatment (21). The PCR rates in the present study were $50.4 \%$ in the training cohort and $37.5 \%$ in the validation cohort, which was consistent with those of several previous studies (30). Depending on these findings, we speculate that some patients could obtain PCR after TACE, for whom close follow-up without immediately sequential TACE or operation is enough. It is of great clinical significance to identify these patients and avoid overtreatment. Further multi-center and randomized controlled trials are needed to confirm this hypothesis.

Some limitations in this study should be considered. First, the limited sample size may weaken the statistical power of this analysis. However, to our knowledge, there is just a very small proportion of BCLC stage A patients that have received preoperative TACE. Second, the patients in our study were from different centers, which made it difficult to collect the pre-TACE data. The insufficient data did not enable us to analyze the variation of some characteristics, such as AFP, HBV-DNA and maximum tumor diameter, before and after TACE treatment. Third, TACE procedures may vary across different centers despite complying with relevant guidelines. Finally, because most of the patients in our study had HBV infection, our nomogram requires further validation in areas with different underlying etiologies of HCC worldwide.

In conclusion, by incorporating 5 post-TACE predictors of PCR, we constructed an easy-to-use and accurate nomogram. The nomogram can estimate the possibility of PCR after TACE in patients with HCC, guide the subsequent treatment and eventually improve clinical outcomes.

\section{Acknowledgments}

Funding: None.

\section{Footnote}

Reporting Checklist: The authors have completed the TRIPOD reporting checklist. Available at https://dx.doi. org/10.21037/atm-21-1120

Data Sharing Statement: Available at https://dx.doi. org/10.21037/atm-21-1120

Conflicts of Interest: All authors have completed the ICMJE uniform disclosure form (available at https://dx.doi. org/10.21037/atm-21-1120). The authors have no conflicts of interest to declare.

Ethical Statement: The authors are accountable for all aspects of the work in ensuring that questions related to the accuracy or integrity of any part of the work are appropriately investigated and resolved. The study was conducted in accordance with the Declaration of Helsinki (as revised in 2013). The study was approved by the institutional ethics board of Eastern Hepatobiliary Surgery 
Hospital (No.: EHBHKY2020-K-028) and by the ethical review board of Mengchao Hepatobiliary Hospital of Fujian Medical University (No.: 2019-063-01). Informed consent was taken from all the patients.

Open Access Statement: This is an Open Access article distributed in accordance with the Creative Commons Attribution-NonCommercial-NoDerivs 4.0 International License (CC BY-NC-ND 4.0), which permits the noncommercial replication and distribution of the article with the strict proviso that no changes or edits are made and the original work is properly cited (including links to both the formal publication through the relevant DOI and the license). See: https://creativecommons.org/licenses/by-nc-nd/4.0/.

\section{References}

1. European Association for the Study of the Liver. Electronic address: easloffice@easloffice.eu; European Association for the Study of the Liver. EASL Clinical Practice Guidelines: Management of hepatocellular carcinoma. J Hepatol 2018;69:182-236.

2. Omata M, Cheng AL, Kokudo N, et al. Asia-Pacific clinical practice guidelines on the management of hepatocellular carcinoma: a 2017 update. Hepatol Int 2017;11:317-70.

3. Heimbach JK, Kulik LM, Finn RS, et al. AASLD guidelines for the treatment of hepatocellular carcinoma. Hepatology 2018;67:358-80.

4. Chen RX, Gan YH, Ge NL, et al. Comparison of transarterial chemoembolization with radiofrequency ablation for unresectable Barcelona Clinic Liver Cancer stage 0/A hepatocellular carcinoma: a propensity score matching. J Gastroenterol Hepatol 2016;31:442-9.

5. Kim BK, Kim SU, Kim KA, et al. Complete response at first chemoembolization is still the most robust predictor for favorable outcome in hepatocellular carcinoma. J Hepatol 2015;62:1304-10.

6. Lencioni R, de Baere T, Soulen MC, et al. Lipiodol transarterial chemoembolization for hepatocellular carcinoma: A systematic review of efficacy and safety data. Hepatology 2016;64:106-16.

7. Miller AB, Hoogstraten B, Staquet M, et al. Reporting results of cancer treatment. Cancer 1981;47:207-14.

8. Lencioni R, Llovet JM. Modified RECIST (mRECIST) assessment for hepatocellular carcinoma. Semin Liver Dis 2010;30:52-60.

9. Kubota K, Hisa N, Nishikawa T, et al. Evaluation of hepatocellular carcinoma after treatment with transcatheter arterial chemoembolization: comparison of Lipiodol-CT, power Doppler sonography, and dynamic MRI. Abdom Imaging 2001;26:184-90.

10. Tezuka M, Hayashi K, Kubota K, et al. Growth rate of locally recurrent hepatocellular carcinoma after transcatheter arterial chemoembolization: comparing the growth rate of locally recurrent tumor with that of primary hepatocellular carcinoma. Dig Dis Sci 2007;52:783-8.

11. Hunt SJ, Yu W, Weintraub J, et al. Radiologic monitoring of hepatocellular carcinoma tumor viability after transhepatic arterial chemoembolization: estimating the accuracy of contrast-enhanced cross-sectional imaging with histopathologic correlation. J Vasc Interv Radiol 2009;20:30-8.

12. Kalb B, Chamsuddin A, Nazzal L, et al. Chemoembolization follow-up of hepatocellular carcinoma with MR imaging: usefulness of evaluating enhancement features on one-month posttherapy MR imaging for predicting residual disease. J Vasc Interv Radiol 2010;21:1396-404.

13. Brown KT, Do RK, Gonen M, et al. Randomized Trial of Hepatic Artery Embolization for Hepatocellular Carcinoma Using Doxorubicin-Eluting Microspheres Compared With Embolization With Microspheres Alone. J Clin Oncol 2016;34:2046-53.

14. Golfieri R, Giampalma E, Renzulli M, et al. Randomised controlled trial of doxorubicin-eluting beads vs conventional chemoembolisation for hepatocellular carcinoma. Br J Cancer 2014;111:255-64.

15. Serenari M, Han KH, Ravaioli F, et al. A nomogram based on liver stiffness predicts postoperative complications in patients with hepatocellular carcinoma. J Hepatol 2020;73:855-62.

16. Shim JH, Han S, Shin YM, et al. Optimal measurement modality and method for evaluation of responses to transarterial chemoembolization of hepatocellular carcinoma based on enhancement criteria. J Vasc Interv Radiol 2013;24:316-25.

17. Bedossa P, Poynard T. An algorithm for the grading of activity in chronic hepatitis $\mathrm{C}$. The METAVIR Cooperative Study Group. Hepatology 1996;24:289-93.

18. Steyerberg EW, Vergouwe Y. Towards better clinical prediction models: seven steps for development and an ABCD for validation. Eur Heart J 2014;35:1925-31.

19. Wang H, Du PC, Wu MC, et al. Postoperative adjuvant transarterial chemoembolization for multinodular hepatocellular carcinoma within the Barcelona Clinic 
Liver Cancer early stage and microvascular invasion. Hepatobiliary Surg Nutr 2018;7:418-28.

20. Sieghart W, Hucke F, Peck-Radosavljevic M. Transarterial chemoembolization: modalities, indication, and patient selection. J Hepatol 2015;62:1187-95.

21. Agopian VG, Morshedi MM, McWilliams J, et al. Complete pathologic response to pretransplant locoregional therapy for hepatocellular carcinoma defines cancer cure after liver transplantation: analysis of 501 consecutively treated patients. Ann Surg 2015;262:536-45; discussion 543-5.

22. Bargellini I, Bozzi E, Campani D, et al. Modified RECIST to assess tumor response after transarterial chemoembolization of hepatocellular carcinoma: CTpathologic correlation in 178 liver explants. Eur J Radiol 2013;82:e212-8.

23. Yu JS, Kim YH, Rofsky NM. Dynamic subtraction magnetic resonance imaging of cirrhotic liver: assessment of high signal intensity lesions on nonenhanced T1weighted images. J Comput Assist Tomogr 2005;29:51-8.

24. Haubold J, Ludwig JM, Li Y, et al. Measuring the density of iodine depositions: Detecting an invisible residual tumor after conventional transarterial chemoembolization. PLoS One 2020;15:e0227972.

25. Miyayama S, Yamashiro M, Hashimoto M, et al. Comparison of local control in transcatheter arterial chemoembolization of hepatocellular carcinoma $\leq 6$ $\mathrm{cm}$ with or without intraprocedural monitoring of the embolized area using cone-beam computed tomography. Cardiovasc Intervent Radiol 2014;37:388-95.

26. Donati OF, Do RK, Hötker AM, et al. Interreader and inter-test agreement in assessing treatment response following transarterial embolization for hepatocellular carcinoma. Eur Radiol 2015;25:2779-88.

27. Peng J, Kang S, Ning Z, et al. Residual convolutional neural network for predicting response of transarterial chemoembolization in hepatocellular carcinoma from CT imaging. Eur Radiol 2020;30:413-24.

28. Allard MA, Sebagh M, Ruiz A, et al. Does pathological response after transarterial chemoembolization for hepatocellular carcinoma in cirrhotic patients with cirrhosis predict outcome after liver resection or transplantation? J Hepatol 2015;63:83-92.

29. Liu G, Ouyang Q, Xia F, et al. Alpha-fetoprotein response following transarterial chemoembolization indicates improved survival for intermediate-stage hepatocellular carcinoma. HPB (Oxford) 2019;21:107-13.

30. Golfieri R, Cappelli A, Cucchetti A, et al. Efficacy of selective transarterial chemoembolization in inducing tumor necrosis in small $(<5 \mathrm{~cm})$ hepatocellular carcinomas. Hepatology 2011;53:1580-9.

31. Vesselle G, Quirier-Leleu C, Velasco S, et al. Predictive factors for complete response of chemoembolization with drug-eluting beads (DEB-TACE) for hepatocellular carcinoma. Eur Radiol 2016;26:1640-8.

32. Jeong SO, Kim EB, Jeong SW, et al. Predictive Factors for Complete Response and Recurrence after Transarterial Chemoembolization in Hepatocellular Carcinoma. Gut Liver 2017;11:409-16.

33. Kim DY, Ryu HJ, Choi JY, et al. Radiological response predicts survival following transarterial chemoembolisation in patients with unresectable hepatocellular carcinoma. Aliment Pharmacol Ther 2012;35:1343-50.

34. Choi J, Shim JH, Shin YM, et al. Clinical significance of the best response during repeated transarterial chemoembolization in the treatment of hepatocellular carcinoma. J Hepatol 2014;60:1212-8.

35. Su YW, Huang YW, Chen SH, et al. Quantitative analysis of plasma HBV DNA for early evaluation of the response to transcatheter arterial embolization for HBVrelated hepatocellular carcinoma. World J Gastroenterol 2005;11:6193-6.

36. Dioguardi Burgio M, Ronot M, Bruno O, et al. Correlation of tumor response on computed tomography with pathological necrosis in hepatocellular carcinoma treated by chemoembolization before liver transplantation. Liver Transpl 2016;22:1491-500.

37. Liao $M$, Zhu Z, Wang $\mathrm{H}$, et al. Adjuvant transarterial chemoembolization for patients after curative resection of hepatocellular carcinoma: a meta-analysis. Scand J Gastroenterol 2017;52:624-34.

38. Yoon JS, Sinn DH, Lee JH, et al. Tumor Marker-Based Definition of the Transarterial ChemoembolizationRefractoriness in Intermediate-Stage Hepatocellular Carcinoma: A Multi-Cohort Study. Cancers (Basel) 2019;11:1721.

39. Ranieri G, Ammendola M, Marech I, et al. Vascular endothelial growth factor and tryptase changes after chemoembolization in hepatocarcinoma patients. World J Gastroenterol 2015;21:6018-25.

40. Iwakiri Y, Groszmann RJ. The hyperdynamic circulation of chronic liver diseases: from the patient to the molecule. Hepatology 2006;43:S121-31.

41. Sun Z, Li G, Ai X, et al. Hepatic and biliary damage after transarterial chemoembolization for malignant hepatic tumors: incidence, diagnosis, treatment, outcome and 
mechanism. Crit Rev Oncol Hematol 2011;79:164-74.

42. Poon RT, Fan ST, Lo CM, et al. Long-term prognosis after resection of hepatocellular carcinoma associated with hepatitis B-related cirrhosis. J Clin Oncol 2000;18:1094-101.

43. Xiao EH, Guo D, Bian DJ. Effect of preoperative transcatheter arterial chemoembolization on angiogenesis of hepatocellular carcinoma cells. World J Gastroenterol 2009;15:4582-6.

44. Luo J, Peng ZW, Guo RP, et al. Hepatic resection versus transarterial lipiodol chemoembolization as the initial treatment for large, multiple, and resectable hepatocellular carcinomas: a prospective nonrandomized analysis. Radiology 2011;259:286-95.

45. Hsu KF, Chu CH, Chan DC, et al. Superselective transarterial chemoembolization vs hepatic resection for resectable early-stage hepatocellular carcinoma in patients with Child-Pugh class a liver function. Eur J Radiol 2012;81:466-71.

46. Guo Z, Zhong Y, Hu B, et al. Hepatic resection or transarterial chemoembolization for hepatocellular carcinoma within Milan criteria: A propensity score matching analysis. Medicine (Baltimore) 2017;96:e8933.

Cite this article as: Lin J, Li X, Shi X, Zhang L, Liu H, Liu J, Wang K, Shen F. Nomogram for predicting pathologic complete response after transarterial chemoembolization in patients with hepatocellular carcinoma. Ann Transl Med 2021;9(14):1130. doi: 10.21037/atm-21-1120 